\title{
Departure Interval Adjustment to Alleviate Bus Bunching under Dynamic Demand and Traffic Condition
}

\author{
Yanjia Fu ${ }^{1}$, Rui Zhao ${ }^{2}$, Wenyi Zhang ${ }^{1, a}$ and Zhongzhu Shang ${ }^{3}$ \\ ${ }^{1}$ MOE Key Laboratory for Urban Transportation Complex Systems Theory and Technology, Beijing \\ Jiaotong University, Beijing 100044, China; \\ ${ }^{2}$ Bestrans Co., Ltd, Beijing 100044, China; \\ ${ }^{3}$ No.1 Engineering Corporation Limited of CR20G, Suzhou 215151, China. \\ awyzhang@bjtu.edu.cn
}

Keywords: green transportation, transit system, bus bunching, dynamic passenger demand.

\begin{abstract}
As a green mode, public transportation makes great contribution in alleviating urban traffic congestion and promoting sustainable urban development. In the process of giving priority to the development of public transport, how to ease or solve the bus bunching is always a hot issue in the field of public transport management research. Most recently, the authors suggested a combined strategy to actively alleviate bus bunching under dynamic traffic condition from a tactical perspective, where, however, the average passenger arrival rate is assumed to be fixed. Seeing that this assumption may be unrealistic, this paper relaxes this assumption and then establishes a mathematical model focusing on departure interval optimization under dynamic passenger demand and traffic condition. A multi-round compass search algorithm is employed to solve the model. Numerical example is conducted to perform the algorithm and management strategy. It is founded that both algorithm and strategy is effective.
\end{abstract}

\section{Introduction}

With the rapid growth of private car ownership and travel demand, atmospheric pollution and city traffic congestion have intensified over the passing years, so the operating environment of the ground transport system has also been deteriorating. Bus bunching, as a result of this, has become a major stumbling block in returning public transport to normal and improving the service standard. Therefore, in the process of giving priority to the development of public transport, how to ease or solve the bus bunching is always a hot issue in the field of public transport management research and has attracted the attention of many relevant scholars [1].

Newell and Potts [2] firstly pointed out the root of bus bunching: if the bus running early encounters disturbance, it will arrive at station later than scheduled time. As a consequence, this bus tends to serve more passengers along the way, which will exacerbate its delay time. As time goes by, the next bus gradually catches up with this bus. Two measures are explored to ease bus bunching: bus holding and inter-station speed adjustment.

Bus holding: Fu and Yang [3] carry on and conduct the research on two kinds of bus holding project and do the simulation separately, one is to calculate stopover time based on the headway with bus ahead, the other is to calculate time considering both headways of the buses in front and behind. Zolfaghari et al. [4] used the real-time position information of the bus on a specific bus line to establish the bus holding mathematical model, aiming at achieving the minimal sum of the passenger waiting time of all bus stations on the line. Daganzo [5] proposed the targeting bus headway theory, when the bus arrives at the control point set in advance, it is necessary to judge the relationship between the actual headway with the bus in front and targeting bus headway. If the actual headway is shorter than the targeting period, this bus needs to stay a while at station. If the opposite happens, the bus is required to depart immediately. John and Donald [6], casting the targeting bus headway theory away, proposed to select the right control point to calculate stationed stopover time in order to achieve the purpose of balancing headway when the interval between stations is uneven caused by traffic flow interference. Liang [7] recommended a self-adaptive holding control strategy to equalize 
bus headway. Recently, Zhang [8] modeled and analyzed holding strategy, stop-skipping strategy and hybrid strategy, according to the real-time bus position and passenger demand flow.

Inter-station speed adjustment: Daganzo and Pilachowski [9] proposed the self-adaptive control method to adjust the bus speed. Ceder et al. [10] applied the speed adjustment between stations method to multiple bus route schedules for real-time regulation, more research and application of speed adjustment method can be found in the Ref. [11].

For large and medium-sized cities in China, the short inter-station distance and frequent congestion make the speed adjustment less available. In contrast, bus holding may be effective. However, the above mentioned literature focuses on real-time control from an operational perspective. This passive adjustment operations are intensive and are difficult to guarantee the effectiveness when encountering severe disturbances. Hammerle et al. [12] reported that irregular travel time and departure frequency should be mainly responsible for bus bunching, especially the latter. Seeing these, Fu et al. [13] recently suggested combing departure interval adjustment and bus holding to actively alleviate bus bunching under dynamic traffic condition from a tactical perspective; however, it was assumed in this study that the average passenger arrival rate is fixed, which may be unrealistic. The main contribution of this paper is to relax this assumption and then establishes a mathematical model focusing on departure interval optimization (since bus holding at crowded city center is not always feasible and may also cause resistance from passengers). It is expected that this study can provide advisable reference to practice.

\section{Mathematical Modeling}

A most recent study of our authors assumed that passengers arrive at a single station in the manner of Poisson distribution with a fixed arrival rate. This assumption may not always be realistic in some circumstances. Therefore, this article relaxes the hypothesis. Suppose that the passenger arrival number of each station obeys the Poisson distribution of the average arrival rate varies with time. Other assumptions include: (1) Buses are of the same type, and operate from the start of the line to the end. (2) There is no waiting for the bus to get into the station. (3) Travel time between two adjacent stations varies with time, and can be expressed as a time-dependent function. This assumption has been widely discussed in vehicle routing problem [14].

Symbols used in this section are listed ahead of time to facilitate the modeling statement.

$N$ is the number of stations indexed by $i \in[1, N]$ which increase from starting point to end point.

$K$ is the number of bus runs (written as buses for brevity) indexed by $k \in[1, K]$.

$s_{k}$ is initially scheduled time for $k$ to arrive at the starting station (i.e., Station 1).

$\alpha_{i}$ is the weight of station $i$ in computing headway, and there is $\sum_{i \in N} \alpha_{i}=1$.

$\beta_{i}$ is the weight of station $i$ in computing passenger flow; and let $\beta_{i}=\gamma \alpha_{i}$, where $\gamma$ is a constant.

$x_{\max }$ is maximum time-adjusting amount of departure time at the start station.

$v_{i}(t)$ is the averaged passenger arrival rate at the station at instant $t$.

$T_{i}(t)$ is the travel time if leaving from station $i$ to $i+1$ at time $t$, where $i \leq N-1$.

$u_{i}^{k}$ is the number of boarding passengers for bus $k$ at station $i$.

$\bar{u}_{i}$ is the average of $u_{i}^{k}$ for all buses at station $i$.

$t_{i}^{k}$ is the time that bus $k$ arrives at station $i$.

$\hat{t}_{i}^{k}$ is the time that bus $k$ leave station $i$.

$\Delta t_{i}^{k, k+1}$ is the difference of arrival time between bus $k+1$ and bus $k$ at station $i$.

$\Delta \bar{t}$ is the average of $\Delta t_{i}^{k, k+1}$ for all stations and buses.

$x_{k}$ is the decision variable denoting the arrival time adjustment amount for bus $k$ at the starting station. 
Based on above assumptions and notations, we present below the model formulation for the current problem.

$$
\begin{aligned}
& \min Z(\mathbf{x})=\sum_{i=1}^{N-1} \sum_{k=1}^{K-1} \alpha_{i}\left(\frac{\Delta t_{i}^{k, k+1}-\Delta \bar{t}}{\Delta \bar{t}}\right)^{2}+\sum_{i=1}^{N-1} \sum_{k=1}^{K} \beta_{i}\left(\frac{\int_{\hat{t}_{i}^{k-1}}^{t_{i}^{k}} v_{i}(t) d t-\bar{u}_{i}}{\bar{u}_{i}}\right)^{2} \\
& \Delta t_{i}^{k, k+1}=\left\{\begin{array}{ll}
x_{k+1}+s_{k+1}-x_{k}-s_{k}, & \text { if } i=1 \\
t_{i}^{k+1}-t_{i}^{k}=\hat{t}_{i-1}^{k+1}-\hat{t}_{i-1}^{k}+T_{i-1}\left(\hat{t}_{i-1}^{k+1}\right)-T_{i-1}\left(\hat{t}_{i-1}^{k}\right), & \text { if } i \geq 2
\end{array} \forall k \leq K-1\right. \\
& \Delta \bar{t}=\frac{1}{(N-1)(K-1)} \sum_{k=1}^{K-1} \sum_{i=1}^{N-1} \Delta t_{i}^{k, k+1} \\
& u_{i}^{k}=\int_{\hat{t}_{i}^{k-1}}^{\hat{t}_{i}^{k}} v_{i}(t) d t \\
& \bar{u}_{i}=\frac{1}{K} \sum_{k=1}^{K} u_{i}^{k} \\
& t_{i}^{k}=\left\{\begin{array}{ll}
x_{k}+s_{k}, & \text { if } i=1 \\
\hat{t}_{i-1}^{k}+T_{i-1}\left(\hat{t}_{i-1}^{k}\right), & \text { if } i \geq 2
\end{array} \quad \forall k \geq 1\right. \\
& \hat{t}_{i}^{k}=\left\{\begin{array}{ll}
t_{i}^{k}+\bar{w}_{i}^{k}, & \text { if } k=1 \\
\max \left\{t_{i}^{k}+\bar{w}_{i}^{k}, \hat{t}_{i}^{k-1}\right\}, & \text { if } k \geq 2
\end{array} \forall i \geq 1\right. \\
& \left|x_{k}\right| \leq x_{\max }, \forall k \leq K \\
& x_{k}+s_{k}<x_{k+1}+s_{k+1}, \forall k \leq K-1
\end{aligned}
$$

The economical meanings of Eqs. (1)-(9) are stated as follows.

Eq. (1) is the objective function, where the first and the second term on the right side formulate the headway and the passenger flow deviation, respectively.

Eqs. (2) and (3) give the calculation formulations for the headways at any station and the averaged headway for this line, respectively. Eqs. (4) and (5) give the calculation formulations for the passenger flows of each bus at any station and the averaged flow of all buses at any station, respectively.

Eqs. (6) and (7) calculate the arrival and the departure time of each bus at any station, respectively. Note that Eq. (7) defines a first-in-first-out rule to regulate the activity of a bus at a station. This rule is also applied in practice by administrators.

Eq. (8) is the adjustment upper bound constraint. Eq. (9) requires that bus that is initially scheduled to arrive at the origin station earlier should also arrive earlier after adjusted.

\section{Solution Algorithm and Numerical Example}

Solution Algorithm. Considering the characteristics of model (i.e., the objective function has no closed-form expression and may not be differentiable), the authors [13] recently established a multiround compass search algorithm (MCSA) to solve the bus bunching model with fixed passenger arrival rate. In contrast to the compass search algorithm (CSA) in Zhang et al. [15], MCSA makes two improvements: one is to introduce a set of (instead of a single) initial guesses; the other is to develop a continuative iteration mechanism. The first one enables MCSA to search within a larger domain, which prevents algorithm from falling in local optimal solutions. The second one requires to repeat CSA many rounds based on the optimal solutions derived from the last round, until no improvement is available; and the final optimal solution is selected as the optimal one among the five. These two revisions grant MCSA a superior performance. Considering the continuity and similarity between the current problem and Fu's problem, MCSA is still applied here to solve the current problem. Due to limited space and the essence of this paper, here we do not list the procedure of MCSA again. For more details regarding MCSA, readers can either refer to Ref. [13] or directly contact the corresponding author. 
In subsequent numerical example, sensitivity analyses with respect to adjustment upper bounds $x_{\max }$ will be made. When using MCSA, five initial guesses are set. If the optimal solution worsens when $x_{\max }$ increases, five new initial guesses based on the optimal solution derived from the smaller $x_{\max }$ will be generated for the larger $x_{\max }$ to re-run MCSA. To name an example, if the optimal solution $\mathbf{x} 1^{*}$ obtained by $x_{\max }=1$ is better than the optimal solution $\mathbf{x} 3^{*}$ obtained by $x_{\max }=3$, then five new initial solutions $\mathbf{x}^{0}=\left\{\mathbf{x} 1^{*}, \mathbf{x} 1^{*} \pm 0.5 \times \mathbf{3}, \mathbf{x} 1^{*} \pm 1 \times 3\right\}$ (Note that $\{\mathrm{a}: \mathrm{b}: \mathrm{c}\}$ is the set of numbers increasing from a to $\mathrm{c}$ with step-size $\mathrm{b}$ ) is generated and substituted into MCSA under $x_{\max }=3$ to identify a superior solution. Such a strategy is also applied in Ref. [13].

Numerical Analysis. In this section, we present a numerical example based on a bus line with ten stations (see Fig. 1) to perform the current model and algorithm.

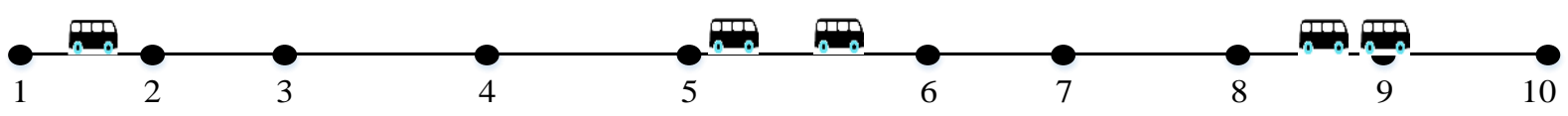

Fig. 1 Bus line schematic

The values of parameters in this numerical example are set as follows: $\alpha=\left(\alpha_{1}, \alpha_{2}, \cdots, \alpha_{i}, \cdots, \alpha_{10}\right)$, $\alpha=(0.120,0.083,0.058,0.061,0.116,0.113,0.184,0.123,0.087,0.054)$ and $\gamma=0.5$.

The research duration for this line ranges from 7:30 to 8:30, and the initial departure headway is 6 minutes. The travel time between two adjacent stations in Appendix A.

Suppose vehicles are of the same type, where passengers get on from the front door and get off from the behind door. For the bus of this type, the boarding time at a station can be computed as follows Ref. [16].

$$
\bar{w}_{i}^{k}=\max \left\{\theta_{1} u_{i}^{k}, \theta_{2} d_{i}^{k}\right\}+w_{0}
$$

where $\theta_{1}$ and $\theta_{2}$ are respectively the marginal time for boarding and alighting per passenger, $d_{i}^{k}$ is number of passengers alighting from the $k$ bus at $i$ station, and $w_{0}$ is the time for door opening and closing. According to Ref. [15], $\theta_{1}=1.78$ seconds, $\theta_{2}=1.48$ seconds, $w_{0}=3.15$ seconds.

Appendix B shows Passenger arrival rate at each station. The number of people getting off is $d_{i}^{k}$ at $i(1 \leq i \leq 9)$ station in the $k$ bus is as shown in Fig. 2(a-c). When the bus arrives at the terminal, all passengers get off.
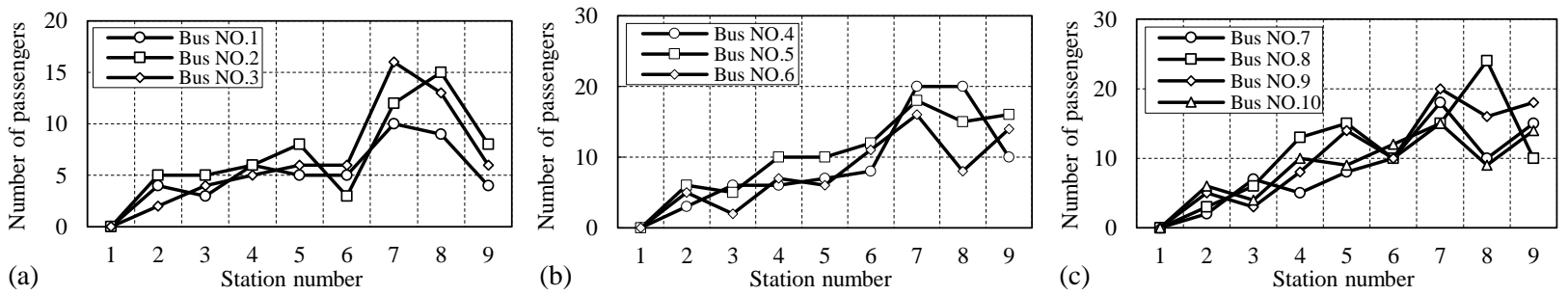

Fig. 2 Number of alighting passengers at each station for all buses: (a) for Bus Nos. 1-3; (b) for Bus Nos. 4-6; and (c) for Bus Nos. 7-10.

Based on the above parameter settings, this section sets three sets of maximum adjustments, i.e. $x_{\max }=\{1,3,5\}$ (unit: $\min$ ), to perform sensitivity analysis on $x_{\max }$. In addition, for each set of parameter combinations, five initial solutions are set for MCSA, i.e., $\mathbf{x}^{\mathbf{0}}=\left\{\mathbf{0}, \mathbf{0} \pm 0.5 \mathbf{x}_{\max }, \mathbf{0} \pm \mathbf{x}_{\max }\right\}$.

When $\lambda_{0}=0.6 x_{\max }$, the objective function obtains the optimal solution. In the optimal solution, the value of the objective function varies with the number of iterations, as shown in Fig. 3. 


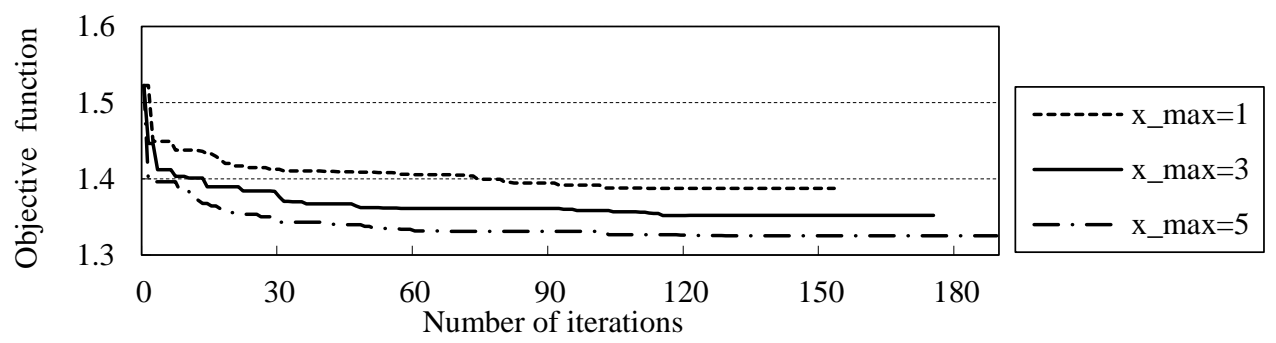

Fig. 3 The evolution of objective function value as iteration proceeds.

When $\lambda_{m+1}=0.9 \lambda_{m}, m=0,1,2 \cdots$, these three groups of maximum adjustments get the value of the objective function, as shown in Fig. 4.

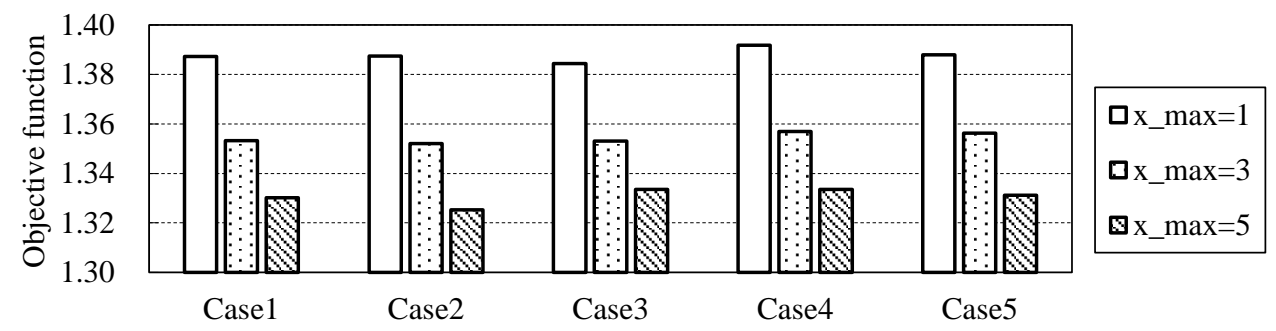

Fig. 4 The optimal objective function values under different parameter scenarios: Case 1 means $\lambda_{0}=0.5 x_{\max }$; Case 2 means $\lambda_{0}=0.6 x_{\max }$; Case 3 means $\lambda_{0}=0.7 x_{\max }$; Case 4 means $\lambda_{0}=0.8 x_{\max }$; Case 5 means $\lambda_{0}=0.9 x_{\max }$.

It can be seen from the Fig.4 that the optimal solution is obtained when $\lambda_{0}=0.6 x_{\max }$, and the objective function value at this time is 1.33 . The optimization result is improved by $13 \%$. In general, when the maximum adjustment amount becomes larger, the search range becomes larger and the objective function value becomes smaller.

The time tables before and after optimization for all buses are shown in following Table 1.

Table 1 Time tables before and after optimization

\begin{tabular}{ccc}
\hline Bus number & Given timetable & Optimized timetable \\
\hline 1 & $7: 36: 00$ & $7: 31: 46$ \\
2 & $7: 42: 00$ & $7: 37: 00$ \\
3 & $7: 48: 00$ & $7: 43: 07$ \\
4 & $7: 54: 00$ & $7: 49: 10$ \\
5 & $8: 00: 00$ & $7: 55: 09$ \\
6 & $8: 06: 00$ & $8: 01: 04$ \\
7 & $8: 12: 00$ & $8: 07: 02$ \\
8 & $8: 18: 00$ & $8: 13: 00$ \\
9 & $8: 24: 00$ & $8: 19: 00$ \\
10 & $8: 30: 00$ & $8: 25: 00$ \\
\hline
\end{tabular}

\section{Conclusions}

This paper establishes a departure interval optimization model to actively resist bus bunching under dynamic passenger demand and traffic condition, where the inter-station travel time is dynamic, and passengers arrive at stations in the manner of Poisson distribution with variable average arrival rate. A direct search method, i.e., MCSA, is introduced and employed to solve the model. A numerical example is conducted to perform the algorithm and management strategy. It is founded that the strategy is effective. It can not only effectively reduce or eliminate occurrence rate of bus bunching, but also help to balance passenger flows across stations. It is hoped that the method suggested in this paper can provide theoretical guidance and decision support for daily management and operation of transit enterprises, and promote the development of green transportation. 


\section{Acknowledgement}

The authors would like to appreciate Shanshan Jiang for her beneficial help in encoding the numerical example. This work is jointly funded by the National Natural Science Foundation of China (91746201, 71601015).

\section{References}

[1] Lu H P. Approaches towards realization of urban green transportation[J]. Urban Transport of China, 2009, 7(6): 23-27.

[2] Newell G F, Potts R B. Maintaining a bus schedule[C]. Australian Road Research Board. 1964: 388-393.

[3] Fu L, Yang X. Design and implementation of bus-holding control strategies with real-time information[J]. Transportation Research Record, 2002 (1791): 6-12.

[4] Zolfaghari S, Azizi N, Jaber M Y. A model for holding strategy in public transit systems with real-time information[J]. International Journal of Transport Management, 2004, 2(2): 99-110.

[5] Daganzo C F. A headway-based approach to eliminate bus bunching: Systematic analysis and comparisons[J]. Transportation Research Part B, 2009, 43(10): 913-921.

[6] Bartholdi III J J, Eisenstein D D. A self-coördinating bus route to resist bus bunching[J]. Transportation Research Part B, 2012, 46(4): 481-491.

[7] Liang S, Zhao S, Lu C, et al. A self-adaptive method to equalize headways: Numerical analysis and comparison[J]. Transportation Research Part B, 2016, 87: 33-43.

[8] Zhang X M. Research on urban regular transit real-time in-station dispatching strategy[D]. Harbin Institute of Technology, 2016.

[9] Daganzo C F, Pilachowski J. Reducing bunching with bus-to-bus cooperation[J]. Transportation Research Part B, 2011, 45(1): 267-277.

[10] Ceder A, Golany B, Tal O. Creating bus timetables with maximal synchronization[J]. Transportation Research Part A, 2001, 35(10): 913-928.

[11] Ibarra-Rojas O J, Delgado F, Giesen R, et al. Planning, operation, and control of bus transport systems: A literature review[J]. Transportation Research Part B, 2015, 77: 38-75.

[12] Hammerle M, Haynes M, Mcneil S. Use of automatic vehicle location and passenger count data to evaluate bus operations[J]. Transportation Research Record, 2005 (1903): 27-34.

[13] Fu Y J, Zhang W Y, Jiang S S, et al. A combined tactical strategy to actively resist bus bunching under dynamic traffic condition[R]. Research Report, Beijing Jiaotong University, 2018.

[14] Gendreau M, Ghiani G, Guerriero E. Time-dependent routing problems: A review[J]. Computers \& Operations Research, 2015, 64: 189-197.

[15] Zhang W Y, Zhang H M, Guan W, et al. Managing day-to-day network traffic evolution via an altering ex-post information release strategy[J]. Journal of Transportation Engineering, 2018, 144(7): 04018028.

[16] Sun L, Tirachini A, Axhausen K W, et al. Models of bus boarding and alighting dynamics[J]. Transportation Research Part A, 2014, 69: 447-460. 


\section{Appendix A.}
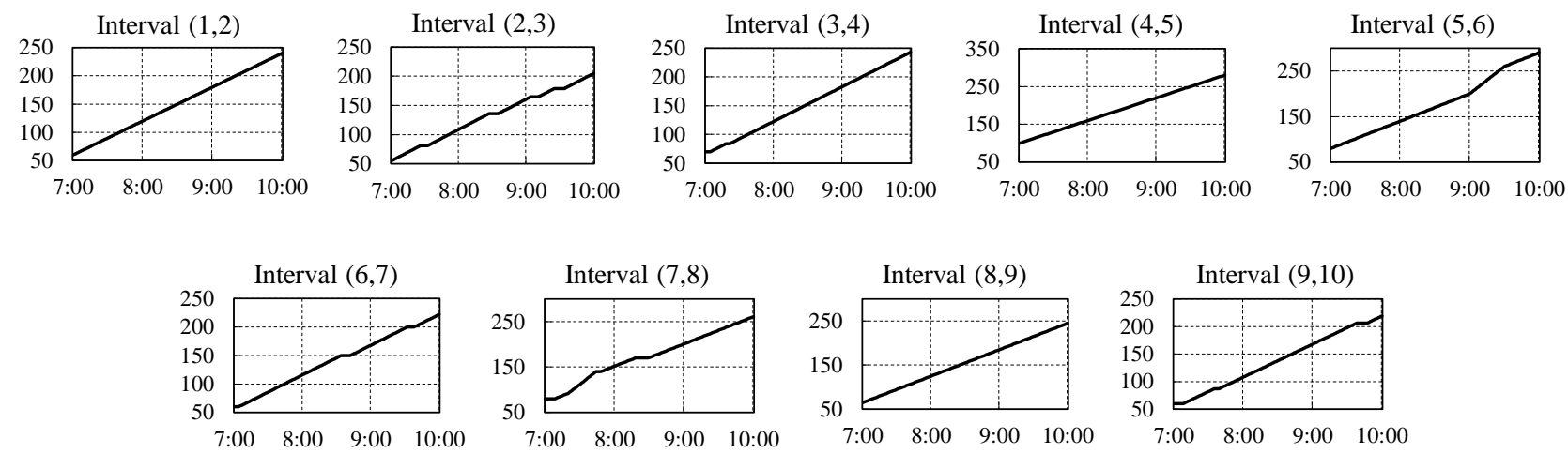

Fig. 5 Inter-station travel time functions. For all subfigures, the title of vertical axe is travel time (unit: second), and the title of horizontal coordinate axis is time.

\section{Appendix B.}
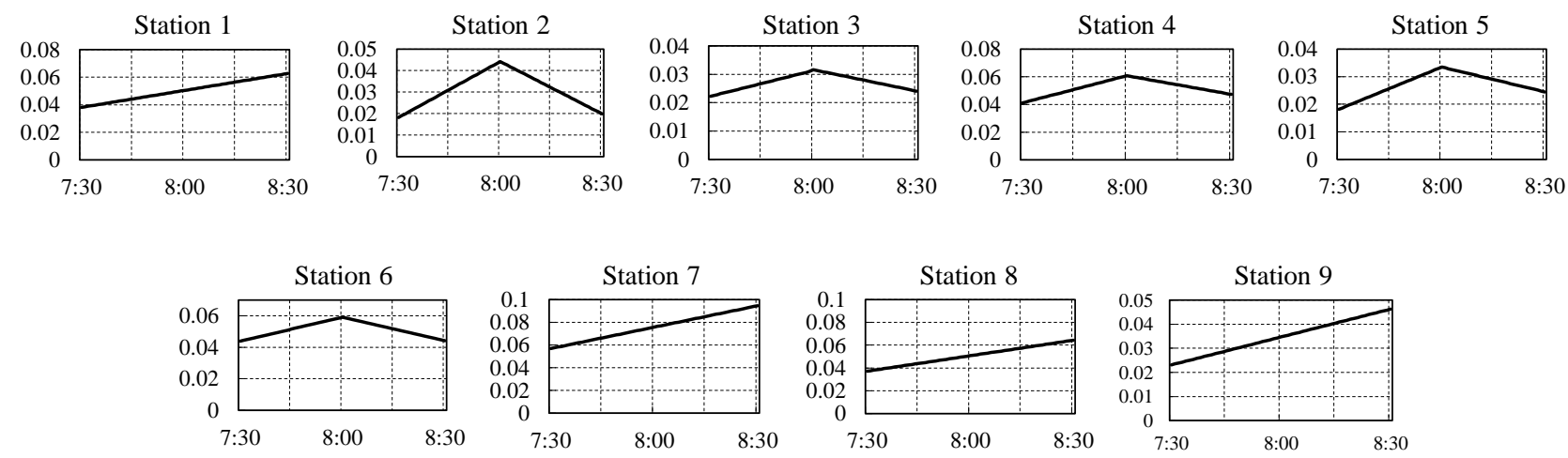

Fig. 6 Passenger arrival rate at stations. For all subfigures, the title of vertical axe is arrival rate (unit:person/second), and the title of horizontal axis is time. 